\title{
Electron Energy Studying of Molecular Structures via Forgotten Topological Index Computation
}

\author{
Wei Gao, ${ }^{1}$ Weifan Wang, ${ }^{2}$ Muhammad Kamran Jamil, ${ }^{3}$ and Mohammad Reza Farahani ${ }^{4}$ \\ ${ }^{1}$ School of Information Science and Technology, Yunnan Normal University, Kunming 650500, China \\ ${ }^{2}$ Department of Mathematics, Zhejiang Normal University, Jinhua 321004, China \\ ${ }^{3}$ Department of Mathematics, Riphah Institute of Computing and Applied Sciences, Riphah International University, \\ 14 Ali Road, Lahore, Pakistan \\ ${ }^{4}$ Department of Applied Mathematics, Iran University of Science and Technology, Tehran 16844, Iran
}

Correspondence should be addressed to Wei Gao; gaowei@ynnu.edu.cn

Received 26 April 2016; Revised 9 July 2016; Accepted 26 July 2016

Academic Editor: Teodorico C. Ramalho

Copyright (C) 2016 Wei Gao et al. This is an open access article distributed under the Creative Commons Attribution License, which permits unrestricted use, distribution, and reproduction in any medium, provided the original work is properly cited.

It is found from the earlier studies that the structure-dependency of total $\pi$-electron energy $E_{\pi}$ heavily relies on the sum of squares of the vertex degrees of the molecular graph. Hence, it provides a measure of the branching of the carbon-atom skeleton. In recent years, the sum of squares of the vertex degrees of the molecular graph has been defined as forgotten topological index which reflects the structure-dependency of total $\pi$-electron energy $E_{\pi}$ and measures the physical-chemical properties of molecular structures. In this paper, in order to research the structure-dependency of total $\pi$-electron energy $E_{\pi}$, we present the forgotten topological index of some important molecular structures from mathematical standpoint. The formulations we obtained here use the approach of edge set dividing, and the conclusions can be applied in physics, chemical, material, and pharmaceutical engineering.

\section{Introduction}

The principal quantum numbers, derived from electrons in an atom or molecule, determine the range of electron energy if we research on the orbital it occupies. "Atomic emission," a very common and interesting phenomenon in atoms, proves to be both the origin of the Fraunhofer lines and an effective approach in this field. To illustrate, it describes that once more energy than minimum for the given situation is stored in one electron, it could be emitted as a photon. Fraunhofer lines are another significant technique which turns out to work pretty well in identification and astronomy, where the measurement of "red shift" in stars exactly comes to the point. As we know, spin and angular momentum can help to intensify the whole energy of an electron, so it can be represented by the set of all of its quantum numbers. To illustrate, the speed that an electron orbits is considered as the electron energy in physics and it could be looked upon as the effect of an electron's energy. But some special phenomena still could occur in the real life and even though two electrons get the same speed, they are not exactly the same. For instance, when two electrons are the same except the spin quantum numbers, they can orbit at the same speed. The case is also likely to happen in two electrons only different in angular momentum numbers with one being a positive number and the other being a negative number.

Having computed within the Huckel tight-binding molecular orbital (HMO) approximation, we get $E_{\pi}$, the total $\pi$-electron energy. As a quantum-chemical property of conjugated molecules, it turns out to be in good accordance to the thermodynamic properties. Based on the eigenvalues of the adjacency matrix of the molecular graph, we can compute out $E_{\pi}$, when the conjugated hydrocarbons are still in their ground electronic states. The mentioned graph can be described like this here: $E_{\pi}=n \alpha+E \beta$. In the graph, $n$ symbolizes the number of carbon atoms; $\alpha$ is the HMO carbon-atom Coulomband; $\beta$ is the carbon-carbon resonance integrals; and for the majority (but not all), conjugated $\pi$-electron systems,

$$
E=\sum_{i=1}^{n}\left|\lambda_{i}\right| .
$$


Here, $\lambda_{1}, \lambda_{2}, \ldots, \lambda_{n}$ represent the eigenvalues of the adjacency matrix $\mathbf{A}$ of the underlying molecular graph $G$, forming the spectrum of $G$.

Referring to the relative researches about $E_{\pi}$ and focusing on the research on its dependence on molecular structure in specific, we find that the term $E$ is the only interesting quantity, and it is defined in (1). As a matter of fact, it is a common and traditional way to regard $E$ as the total $\pi$ electron energy, expressed in $\beta$-units. Hence, there is a need for us to mention that the quantity defined via (1) is called the energy of the graph $G$ in mathematical.

More details on total $\pi$-electron energy can be found in Gutman et al. [1-5], Angelina et al. [6], Türker and Gutman [7], Jones et al. [8], Radenković and Gutman [9], Peric et al. [10], Morales [11], Markovic [12], and Morales [13].

A research on the structure-dependency of total $\pi$ electron energy $E_{\pi}$ in 1972 proposed an approach to the branching of the carbon-atom skeleton by demonstrating that the sum of squares of the vertex degrees of the molecular graph can determine $E_{\pi}$. What is more, it also pointed that $E_{\pi}$ tends to be influenced by the sum of cubes of degrees of vertices of the molecular graph. Indeed, the formulas for total $\pi$-electron energy $E_{\pi}$ also concern the sum of cubes of vertex degrees (in many references, this value can be denoted by $\left.\sum \sigma_{1}^{3}\right)$. In a clear fashion, this quantity is a measure of branching as well.

Thus, in a recent research on the structure-dependency of the total $\pi$-electron energy, it was indicated that another term on which this energy depends is in the form (see Furtula and Gutman [14])

$$
F(G)=\sum_{v \in V(G)} d(v)^{3}=\sum_{u v \in E(G)}\left(d(u)^{2}+d(v)^{2}\right),
$$

where $d(v)$ is denoted as the degree of vertex $v$ (the number of vertex adjacent to vertex $v$ ). In addition, this sum was named forgotten topological index or shortly the $F$-index.

In the web site, http://www.moleculardescriptors.eu/dataset/dataset.htm, the potential ability of the $F$-index was tested using a dataset of octane isomers, which is in accordance to the International Academy of Mathematical Chemistry. In the simplest form, the $F$-index does not recognize heteroatoms and multiple bonds, and this becomes the reason why dataset is chosen as a measure. A list of data including boiling point, melting point, heat capacities, entropy, density, heat of vaporization, enthalpy of formation, motor octane number, molar refraction, acentric factor, total surface area, octanol-water partition coefficient, and molar volume help to compose the octane dataset. The $F$-index shows its strong bonds with most properties here. As a consequence, $F$-index proves to have correlation coefficients greater than 0.95 in entropy and acentric factor.

However, for many other physicochemical characteristics, $F$-index may not be fully correlated. In order to strengthen the predictive ability of $F$-index in potential chemical applications, a linear framework was proposed as follows (see Furtula and Gutman [14]):

$$
\sum_{u v \in E(G)}(d(u)+d(v))+\lambda \sum_{u v \in E(G)}\left(d(u)^{2}+d(v)^{2}\right),
$$

where $\lambda$ is an adaptive parameter which can be adjusted according to the detailed applications in chemical or pharmacy engineering (generally speaking, $\lambda$ always take value from -20 to 20); the first term $\sum_{u v \in E(G)}(d(u)+d(v))$ was defined as the first Zagreb index which was one of the most traditional indexes in chemical science. By means of a large number of experimental studies, this framework can be used in each of the physicochemical properties with fixed octane database. As an example, an evident improvement can be obtained in the octanol-water partition coefficient, and it was pointed out that the absolute value of the correlation coefficient gets a tight maximum if taking $\lambda=-0.14$ in the above framework. Then, by virtue of derivation, the octanolwater partition coefficient of octanes can be stated as the following representation:

$$
\begin{aligned}
& \log P=-0.2058\left(\sum_{u v \in E(G)}(d(u)+d(v))\right. \\
& \left.-0.14 \sum_{u v \in E(G)}\left(d(u)^{2}+d(v)^{2}\right)\right)+7.5864
\end{aligned}
$$

where $\log P$ is the $\operatorname{logarithm}$ function of the octanol-water partition coefficient. This fact implies that the error of mean absolute percentage is only $0.06 \%$ and the correlation coefficient can reach to 0.99896 .

In real engineering implements, the model $\sum_{u v \in E(G)}(d(u)+$ $d(v))+\lambda \sum_{u v \in E(G)}\left(d(u)^{2}+d(v)^{2}\right)$ can be regarded as a generalized framework. For different chemical applications, adaptive parameter $\lambda$ is a key factor which can be changed to the optimal value according to the detailed applications. In the above example, $\lambda$ takes -0.14 , while for other applications $\lambda$ can take other values and it is determined by detailed physical-chemical properties and measured in the chemical experiments.

In the whole article, the molecular structure is modeled as a graph $G$ with vertex set $V(G)$ and edge set $E(G)$, where each vertex represents an atom and each edge denotes a chemical bond between two atoms. A topological index defined on the molecular graph can be considered as a real-valued function $f: G \rightarrow \mathbb{R}^{+}$which maps each chemical structure to a real score. Except the forgotten topological index, there are several famous indices introduced and applied in chemical engineering, such as Wiener index, harmonic index, sum connectivity index, and eccentric index (see Gao et al. [1519], Yang et al. [20], Marana et al. [21], and Li et al. [22] for more details). The terminologies and notations used but not clearly defined in our paper can be found in Bondy and Mutry [23].

Although there have been many advances in degreebased and distance-based indices of molecular graphs, the studies of forgotten topological index for special chemical molecular structures are still largely limited. For this reason, we give the exact expressions of forgotten topological index for several chemical molecular structures which commonly appeared in various chemical environments. 


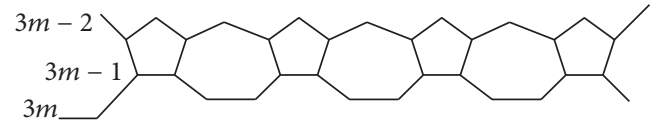

Figure 1: $i$ th period of $\mathrm{SC}_{5} \mathrm{C}_{7}[p, q]$ nanotube.

The rest of the context is arranged as follows: first, we present the forgotten topological index of some nanostructures: $\mathrm{SC}_{5} \mathrm{C}_{7}[p, q]$ nanotubes, $\mathrm{H}$-naphtalenic nanotubes NPHX $[m, n], \mathrm{TUC}_{4}[m, n]$ nanotubes, PAMAM dendrimers: $\mathrm{PD}_{1}[n], \mathrm{PD}_{2}[n]$, and $\mathrm{DS}_{1}[n], \mathrm{VC}_{5} \mathrm{C}_{7}[p, q]$, and $\mathrm{HC}_{5} \mathrm{C}_{7}[p, q]$ nanotubes, zigzag $\operatorname{TUZC}_{6}[m, n]$, and armchair $\operatorname{TUAC}_{6}[m, n]$ nanotubes; second, we calculate the forgotten topological index of several kinds of polyomino chains.

The main trick in our article to deduce the expected result is edge set dividing technology. Let $\delta(G)$ and $\Delta(G)$ be the minimum and maximum degree of $G$, respectively. The edge set $E(G)$ can be divided into several partitions: for any $i$, $2 \delta(G) \leq i \leq 2 \Delta(G)$, let $E_{i}=\{e=u v \in E(G) \mid d(v)+d(u)=i\}$; for any $j,(\delta(G))^{2} \leq j \leq(\Delta(G))^{2}$, let $E_{j}^{*}=\{e=u v \epsilon$ $E(G) \mid d(v) d(u)=j\}$. Using this dividing, the $F$-index can be expressed as

$$
\begin{aligned}
F(G) & =\sum_{E_{i}} \sum_{u v \in E_{i}}\left(d(u)^{2}+d(v)^{2}\right) \\
& =\sum_{E_{j}^{*}} \sum_{u v \in E_{j}^{*}}\left(d(u)^{2}+d(v)^{2}\right) .
\end{aligned}
$$

More specifically, if we denote $E_{i j}=\{e=u v \in E(G) \mid d(v)=$ $i, d(u)=j\}$, then the $F$-index can be further stated as

$$
F(G)=\sum_{E_{i j}}\left|E_{i j}\right|\left(i^{2}+j^{2}\right) .
$$

In view of this alternation and the detailed analysis of molecular structures, the $F$-index of special chemical graphs can be determined.

\section{Forgotten Topological Index of Nanotubes}

In the field of nanomaterial and nanotechnology, there are a large number of new nanostructures being discovered each year. It needs more chemical experiments to figure out their biochemical properties. In this section, we focus on the nanostructures and present the forgotten topological index of some special kinds of nanorelated molecular graphs.

2.1. Forgotten Topological Index of $S_{5} C_{7}[p, q]$ Nanotubes and $\mathrm{H}$-Naphtalenic Nanotubes. In nanoscience, $\mathrm{SC}_{5} \mathrm{C}_{7}[p, q]$ (where $p$ and $q$ express the number of heptagons in each row and the number of periods in whole lattice, resp.) nanotube is a class of $\mathrm{C}_{5} \mathrm{C}_{7}$-net which is yielded by alternating $\mathrm{C}_{5}$ and $\mathrm{C}_{7}$. The standard tiling of $\mathrm{C}_{5}$ and $\mathrm{C}_{7}$ can cover either a cylinder or a torus, and each period of $\mathrm{SC}_{5} \mathrm{C}_{7}[p, q]$ consisted of three rows (more details on $i$ th period can be referred to in Figure 1).

H-Naphtalenic nanotubes $\operatorname{NPHX}[m, n]$ (where $m$ and $n$ are denoted as the number of pairs of hexagons in first row and the number of alternative hexagons in a column, resp.) are a trivalent decoration with sequence of $\mathrm{C}_{6}, \mathrm{C}_{6}, \mathrm{C}_{4}, \mathrm{C}_{6}, \mathrm{C}_{6}, \mathrm{C}_{4}, \ldots$ in the first row and a sequence of $\mathrm{C}_{6}, \mathrm{C}_{8}, \mathrm{C}_{6}, \mathrm{C}_{8}, \ldots$ in the other rows. In other words, this nanolattice can be considered as a plane tiling of $\mathrm{C}_{4}, \mathrm{C}_{6}$, and $\mathrm{C}_{8}$. Therefore, this class of tiling can cover either a cylinder or a torus.

Now, our first result on the forgotten topological index of $\mathrm{SC}_{5} \mathrm{C}_{7}[p, q]$ nanotubes and $\mathrm{H}$-naphtalenic nanotubes is stated as follows.

Theorem 1. One has

$$
\begin{aligned}
& F\left(\mathrm{SC}_{5} \mathrm{C}_{7}[p, q]\right)=216 p q-76 p \\
& F(\operatorname{NPHX}[m, n])=270 m n-76 m
\end{aligned}
$$

Proof. According to the molecular graph structure $\mathrm{SC}_{5} \mathrm{C}_{7}[p, q]$, we see that $\left|E\left(\mathrm{SC}_{5} \mathrm{C}_{7}[p, q]\right)\right|=12 p q-2 p$ and its edge set can be separated into three subsets:

(i) $E_{4}\left(\right.$ or $\left.E_{4}^{*}\right), d(u)=d(v)=2,\left|E_{4}\right|=\left|E_{4}^{*}\right|=p$.

(ii) $E_{6}\left(\right.$ or $\left.E_{9}^{*}\right), d(u)=d(v)=3,\left|E_{6}\right|=\left|E_{9}^{*}\right|=12 p q-9 p$.

(iii) $E_{5}\left(\right.$ or $\left.E_{6}^{*}\right), d(u)=2, d(v)=3$, and $\left|E_{5}\right|=\left|E_{6}^{*}\right|=6 p$.

For H-naphtalenic nanotubes NPHX $[m, n]$, we check that $|E(\mathrm{NPHX}[m, n])|=15 m n-2 m$ and its edge set can be divided into two partitions:

(i) $E_{6}\left(\right.$ or $\left.E_{9}^{*}\right), d(u)=d(v)=3,\left|E_{6}\right|=\left|E_{9}^{*}\right|=15 m n-$ $10 \mathrm{~m}$.

(ii) $E_{5}\left(\right.$ or $\left.E_{6}^{*}\right), d(u)=2, d(v)=3,\left|E_{5}\right|=\left|E_{6}^{*}\right|=8 m$.

At last, we get the desired formulations in terms of the definitions of forgotten topological index.

2.2. Forgotten Topological Index of $\operatorname{TUC}_{4}[m, n]$ Nanotubes and PAMAM Dendrimers. In the nanoscience, $\mathrm{TUC}_{4}[m, n]$ nanotubes (where $m$ and $n$ are denoted as the number of squares in a row and the number of squares in a column, resp.) are plane tiling of $\mathrm{C}_{4}$. This tessellation of $\mathrm{C}_{4}$ can cover either a torus or a cylinder. The $3 \mathrm{D}$ representation of $\mathrm{TUC}_{4}[6, n]$ is described in Figure 2.

Using the trick of edge set dividing, we yield the following statement.

Theorem 2. One has

$$
F\left(\mathrm{TUC}_{4}[m, n]\right)=64 m n-10 m .
$$

Proof. By observing the structure of $\mathrm{TUC}_{4}[m, n]$, we verify that $\left|E\left(\mathrm{TUC}_{4}[m, n]\right)\right|=2 m n+m$ and its edge set can be divided into three partitions:

(i) $E_{6}\left(\right.$ or $\left.E_{9}^{*}\right), d(u)=d(v)=3,\left|E_{6}\right|=\left|E_{9}^{*}\right|=2 m$.

(ii) $E_{7}\left(\right.$ or $\left.E_{12}^{*}\right), d(u)=3, d(v)=4,\left|E_{7}\right|=\left|E_{12}^{*}\right|=2 m$.

(iii) $E_{8}\left(\right.$ or $\left.E_{16}^{*}\right), d(u)=d(v)=4,\left|E_{8}\right|=\left|E_{16}^{*}\right|=m(2 n-3)$.

Therefore, the expected formulation is followed by the definitions of forgotten topological index. 


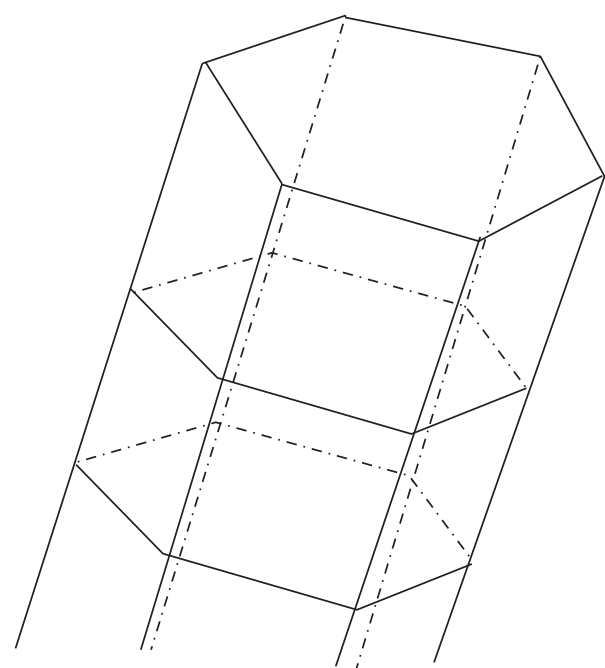

FIGURE 2: The 3D expression of $\mathrm{TUC}_{4}[6, n]$.

Now, we use $\mathrm{PD}_{1}$ to denote PAMAM dendrimers with trifunctional core unit generated by dendrimer generations $G_{n}$ with $n$ growth stages, use $\mathrm{PD}_{2}$ to denote PAMAM dendrimer with different core generated by dendrimer generations $G_{n}$ with $n$ growth stages, and use $\mathrm{DS}_{1}$ to express other kinds of PAMAM dendrimer with $n$ growth stages.

Theorem 3. One has

$$
\begin{aligned}
& F\left(\mathrm{PD}_{1}\right)=492 \cdot 2^{n}-258 \\
& F\left(\mathrm{PD}_{2}\right)=656 \cdot 2^{n}-310 \\
& F\left(\mathrm{DS}_{1}\right)=228 \cdot 3^{n}-160 .
\end{aligned}
$$

Proof. By observation of PAMAM dendrimer $\mathrm{PD}_{1}, \mathrm{PD}_{2}$, and $\mathrm{DS}_{1}$, we ensure that the edge set of $\mathrm{PD}_{1}$ can be divided into four partitions:

(i) $E_{3}\left(\right.$ or $\left.E_{2}^{*}\right), d(u)=1, d(v)=2,\left|E_{3}\right|=\left|E_{2}^{*}\right|=3 \cdot 2^{n}$.

(ii) $E_{3}^{*}, d(u)=1, d(v)=3,\left|E_{3}^{*}\right|=6 \cdot 2^{n}-3$.

(iii) $E_{4}^{*}, d(u)=d(v)=2,\left|E_{4}^{*}\right|=18 \cdot 2^{n}-9$.

(iv) $E_{5}\left(\right.$ or $\left.E_{6}^{*}\right), d(u)=2, d(v)=3,\left|E_{5}\right|=\left|E_{6}^{*}\right|=21 \cdot 2^{n}-$ 12.

The set $E\left(\mathrm{PD}_{2}\right)$ can be divided into four subsets.

(i) $E_{3}\left(\right.$ or $\left.E_{2}^{*}\right), d(u)=1, d(v)=2,\left|E_{3}\right|=\left|E_{2}^{*}\right|=4 \cdot 2^{n}$.

(ii) $E_{3}^{*}, d(u)=1, d(v)=3,\left|E_{3}^{*}\right|=8 \cdot 2^{n}-4$.

(iii) $E_{4}^{*}, d(u)=d(v)=2,\left|E_{4}^{*}\right|=24 \cdot 2^{n}-11$.

(iv) $E_{5}\left(\right.$ or $\left.E_{6}^{*}\right), d(u)=2, d(v)=3,\left|E_{5}\right|=\left|E_{6}^{*}\right|=28 \cdot 2^{n}-$ 14.

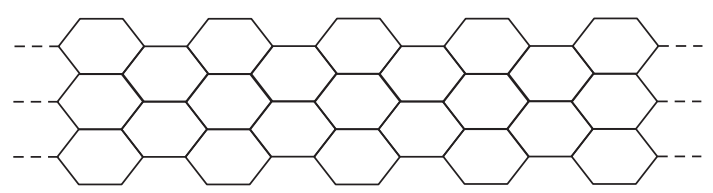

Figure 3: The 3D lattice of the zigzag TUZC $_{6}[10,7]$.

Moreover, the edge set of $\mathrm{DS}_{1}$ can be divided into three parts.

(i) $E_{5}, d(u)=1, d(v)=4,\left|E_{5}\right|=4 \cdot 3^{n}$.

(ii) $E_{4}, d(u)=d(v)=2,\left|E_{4}\right|=10 \cdot 3^{n}-10$.

(iii) $E_{6}\left(\right.$ or $\left.E_{8}^{*}\right), d(u)=2, d(v)=4,\left|E_{6}\right|=\left|E_{8}^{*}\right|=4 \cdot 3^{n}-4$.

Finally, the results are deduced by means of the definitions of forgotten topological index.

2.3. Forgotten Topological Index of $\operatorname{VC}_{5} C_{7}[p, q]$ and $\mathrm{HC}_{5} \mathrm{C}_{7}[p, q]$ Nanotubes. The aim of this subsection is to compute the forgotten topological index of $\mathrm{VC}_{5} \mathrm{C}_{7}[p, q]$ and $\mathrm{HC}_{5} \mathrm{C}_{7}[p, q]$ (where $p$ is the number of pentagons in the first row and $q$ is the number of repetitions in four first rows of vertices and edges) nanotubes. The molecular structures of these nanotubes consist of cycles $C_{5}$ and $C_{7}\left(C_{5} C_{7}\right.$ net which is a trivalent decoration constructed by alternating $\mathrm{C}_{5}$ and $\mathrm{C}_{7}$ ) by different compound. It can cover either a cylinder or a torus.

Now, we present the main results in this subsection.

Theorem 4. One has

$$
\begin{aligned}
& F\left(\mathrm{VC}_{5} \mathrm{C}_{7}[p, q]\right)=432 p q+48 p \\
& F\left(\mathrm{HC}_{5} \mathrm{C}_{7}[p, q]\right)=216 p q+40 p .
\end{aligned}
$$

Proof. By graph analysis and computation, we have $\left|E\left(\mathrm{VC}_{5} \mathrm{C}_{7}[p, q]\right)\right|=24 p q+6 p$ and $\left|E\left(\mathrm{HC}_{5} \mathrm{C}_{7}[p, q]\right)\right|=$ $12 p q+5 p$. Furthermore, we yield two partitions of edge set $E\left(\mathrm{VC}_{5} \mathrm{C}_{7}[p, q]\right): E_{6}, d(u)=d(v)=3 ; E_{5}, d(u)=2$ and $d(v)=3$; and three partitions of edge set $E\left(\mathrm{HC}_{5} \mathrm{C}_{7}[p, q]\right)$ : $E_{4}, d(u)=d(v)=2 ; E_{6}, d(u)=d(v)=3 ; E_{5}, d(u)=2$ and $d(v)=3$.

Consider nanotubes $\mathrm{VC}_{5} \mathrm{C}_{7}[p, q]$ with any $p, q \in \mathbb{N}$; we get $\left|E_{5}\right|=\left|E_{6}^{*}\right|=12 p$ and $\left|E_{6}\right|=\left|E_{9}^{*}\right|=24 p q-6 p$. For nanotube $\mathrm{HC}_{5} \mathrm{C}_{7}[p, q]$ with any $p, q \in \mathbb{N}$, we deduce $\left|E_{4}\right|=$ $\left|E_{4}^{*}\right|=p,\left|E_{5}\right|=\left|E_{6}^{*}\right|=8 p$, and $\left|E_{6}\right|=\left|E_{9}^{*}\right|=12 p q-4 p$.

Therefore, in terms of definitions of the forgotten topological index, we get the final conclusion as expected.

2.4. Forgotten Topological Index of Two Classes of Polyhex Nanotubes. As the last part of this section, we aim to study the forgotten topological index of two classes of polyhex nanotubes: zigzag $\operatorname{TUZC}_{6}[m, n]$ and armchair $\operatorname{TUAC}_{6}[m, n]$, where $m$ is the number of hexagons in the first row and $n$ is the number of hexagons in the first column. The molecular structures of $\mathrm{TUZC}_{6}[m, n]$ and $\mathrm{TUAC}_{6}[m, n]$ can be referred to in Figures 3 and 4, respectively.

Now, we present the main results in this section. 


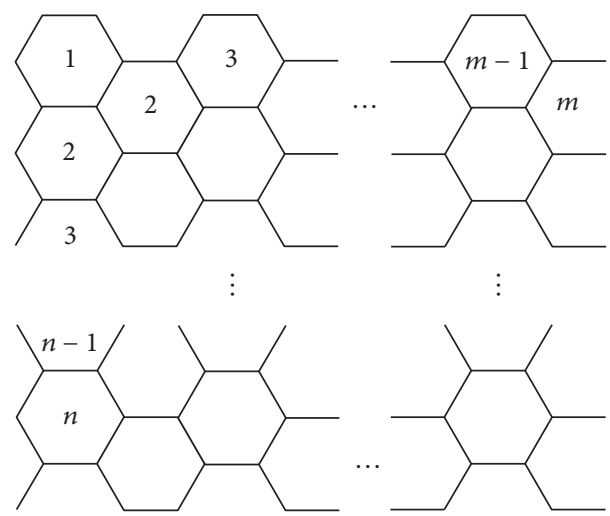

FIgURE 4: The 3D lattice of the armchair $\operatorname{TUAC}_{6}[m, n]$.

Theorem 5. One has

$$
\begin{aligned}
& F\left(\text { TUZC }_{6}[m, n]\right)=54 m n+16 m, \\
& F\left(\text { TUAC }_{6}[m, n]\right)=54 m n+16 m .
\end{aligned}
$$

Proof. It is not hard to check that $\mid E\left(\right.$ TUZC $\left._{6}[m, n]\right) \mid=$ $\mid E\left(\right.$ TUAC $\left._{6}[m, n]\right) \mid=3 m n+2 m$. Consider nanotubes TUZC $_{6}[m, n]$ with any $m, n \in \mathbb{N}$; we deduce that its edge set can be divided into two parts:

(i) $E_{6}\left(\right.$ or $\left.E_{9}^{*}\right), d(u)=d(v)=3$ and $\left|E_{6}\right|=\left|E_{9}^{*}\right|=3 m n-$ $2 m$.

(ii) $E_{5}\left(\right.$ or $\left.E_{6}^{*}\right), d(u)=2, d(v)=3$, and $\left|E_{5}\right|=\left|E_{6}^{*}\right|=4 m$.

For nanotubes $\operatorname{TUAC}_{6}[m, n]$ with any $m, n \in \mathbb{N}$, we find that its edge set can be divided into three parts:

(i) $E_{4}\left(\right.$ or $\left.E_{4}^{*}\right), d(u)=d(v)=2$, and $\left|E_{4}\right|=\left|E_{4}^{*}\right|=m$.

(ii) $E_{6}\left(\right.$ or $\left.E_{9}^{*}\right), d(u)=d(v)=3$, and $\left|E_{6}\right|=\left|E_{9}^{*}\right|=3 m n-$ $m$.

(iii) $E_{5}\left(\right.$ or $\left.E_{6}^{*}\right), d(u)=2, d(v)=3$, and $\left|E_{5}\right|=\left|E_{6}^{*}\right|=$ $2 m$.

Therefore, the conclusion is deduced in view of the definition of forgotten topological index.

\section{Forgotten Topological Index of Polyomino Chains}

From the geometric point of view, a polyomino system is a finite 2-connected plane graph in which each interior cell is encircled by a regular square. In other words, it is an edge-connected union of cells in the planar square lattice. Polyomino chain is a particular polyomino system such that the joining of the centers ( $\operatorname{set} c_{i}$ as the center of the $i$ th square) of its adjacent regular composes a path $c_{1}, c_{2}, \ldots, c_{n}$. Let $\mathbf{B}_{n}$ be the set of polyomino chains with $n$ squares. There is $3 n+1$ edges in every $B_{n} \in \mathbf{B}_{n}$, where $B_{n}$ is named as a linear chain and denoted by $L_{n}$ if the subgraph of $B_{n}$ induced by the vertices with $d(v)=3$ is a molecular graph with exactly $n-2$ squares. Also, $B_{n}$ can be called a zigzag chain and labelled as $Z_{n}$ if the subgraph of $B_{n}$ is induced by the vertices with $d(v)>2$ is $P_{n}$.

The angularly connected squares or branched is a kink of a polyomino chain. A maximal linear chain (containing the terminal squares and kinks at its end) in the polyomino chains is called a segment of polyomino chain. Let $l(S)$ be the length of $S$ which is calculated by the number of squares in $S$. For any segment $S$ of a polyomino chain, we get $l(S) \in$ $[2, \ldots, n]$. Furthermore, we deduce $l_{1}=n$ and $m=1$ for a linear chain $L_{n}$ with $n$ squares and $l_{i}=2$ and $m=n-1$ for a zigzag chain $Z_{n}$ with $n$ squares.

In what follows, we always assume that a polyomino chain consists of a sequence of segments $S_{1}, S_{2}, \ldots, S_{m}$ and $l\left(S_{i}\right)=l_{i}$, where $m \geq 1$ and $i \in\{1,2, \ldots, m\}$. We derive that $\sum_{i=1}^{m} l_{i}=$ $n+m-1$.

The theorems presented in the following can deduce the expression of forgotten topological index of polyomino chains.

Theorem 6. Let $L_{n}, Z_{n}$ be the polyomino chains explained above. One gets

$$
\begin{aligned}
& F\left(L_{n}\right)=54 n-22, \\
& F\left(Z_{n}\right)= \begin{cases}32, & n=1, \\
72 n-58, & n \geq 2 .\end{cases}
\end{aligned}
$$

Proof. For $n=1$, we can check the results directly. In the following consideration, we always suppose that $n \geq 2$.

It is not hard to verify that $\left|E\left(L_{n}\right)\right|=\left|E\left(Z_{n}\right)\right|=3 n+1$. Let

$$
n_{i j}=|\{(u, v) \mid d(u)=i, d(v)=j\}| .
$$

(i) For the polyomino chain $L_{n}$, we have $n_{22}=2, n_{23}=4$, and $n_{33}=3 n-5$.

(ii) For zigzag chain $Z_{n}$, we obtain $n_{22}=n_{34}=2, n_{23}=4$, $n_{24}=2(m-1)$, and $n_{44}=3 n-2 m-5$.

Using $m=n-1$ for $Z_{n}$ and the definition of forgotten topological index, we yield the desired results.

Theorem 7. Let $B_{n}^{1}(n \geq 3)$ be a polyomino chain with $n$ squares and of $m$ segments $S_{1} ; S_{2}$ satisfy $l_{1}=2$ and $l_{2}=n-1$. Then, one has

$$
F\left(B_{n}^{1}\right)=54 n-4 .
$$

Proof. It is trivial for $n=3$; we omit the detailed proof here. For $n \geq 4$, we get $n_{22}=2, n_{23}=5, n_{24}=1, n_{34}=3$, and $n_{33}=$ $3 n-10$. Therefore, by virtue of the definition of forgotten topological index, the desired result is obtained.

We assume that $2 \leq l(i) \leq n-1$ with $1 \leq i \leq m$ in the following consideration of this section.

Theorem 8. Let $B_{n}^{2}(n \geq 4)$ be a polyomino chain with $n$ squares and $m$ segments $S_{1}, S_{2}, \ldots, S_{m}(m \geq 3)$ satisfy $l_{1}=l_{m}=$ 2 and $l_{2}, \ldots, l_{m-1} \geq 3$. Then, one infers

$$
F\left(B_{n}^{2}\right)=54 n+18 m-40 .
$$


Proof. For $B_{n}^{2}$ with $n \geq 4$, we yield $n_{22}=2, n_{23}=2 m, n_{24}=2$, $n_{34}=4 m-6$, and $n_{33}=3 n-6 m+3$. Hence, according to the definition of forgotten topological index, we derive the desired results.

In a similar way, we deduce the following last two conclusions in our paper.

Theorem 9. Let $B_{n}^{3}$ be a polyomino chain with $n$ squares and $m$ segments $S_{1}, S_{2}, \ldots, S_{m}(m \geq 3)$ satisfy $l_{1}=2, l_{2}, \ldots, l_{m-1}$, $l_{m} \geq 3$, or $l_{m}=2, l_{1}, l_{2}, \ldots, l_{m-1} \geq 3$. Then, one obtains

$$
F\left(B_{n}^{3}\right)=18 m+54 n-40 \text {. }
$$

Theorem 10. Let $B_{n}^{4}$ be a polyomino chain with $n$ squares and $m$ segments $S_{1}, S_{2}, \ldots, S_{m}(m \geq 3)$ meet $l_{i} \geq 3(i \in$ $\{1,2, \ldots, m\})$. Then, one has

$$
F\left(B_{n}^{4}\right)=18 m+54 n-40 .
$$

Remark 11. After the manuscript was in press, we received a comment from Akbar Ali. It pointed that Theorems 6-10 can be obtained from Theorem 3.1 in Ali et al. [24]. By comparing Theorem 3.1 and our results Theorems 6-10, we found that the results presented in our paper set the different parameter from the result in Ali et al. [24]. More importantly, the tricks used in our paper are completely different from the former one. All the results yielded in our paper in light of edge set dividing which raised the edge classification in detail. From this point of view, the conclusions Theorems 6-10 are still valuable to the readers.

\section{Conclusion}

In this paper, by means of analyzing of molecular graph structure, edge set dividing approach, and mathematical derivation, we report the forgotten topological index of certain important and widely appeared chemical structures such as $\mathrm{SC}_{5} \mathrm{C}_{7}[p, q]$ nanotubes, $\mathrm{H}$-naphtalenic nanotubes $\mathrm{NPHX}[m, n], \mathrm{TUC}_{4}[m, n]$ nanotubes, and some classes of polyomino chains. These theoretical results achieved in our paper reveal the structure-dependency characteristic of total $\pi$-electron energy $E_{\pi}$ for these molecular structures and illustrate the promising prospects of chemical pharmaceuticals and materials engineering applications such as octanol-water partition coefficient measure and the test of melting point boiling point.

Regrettably, due to the lack of equipment and other experimental conditions, we did not present a specific chemical experiment to show how to apply the theoretical results to specific chemical engineering, whereas we believe the results obtained in this paper can provide theoretical support for the chemical, pharmaceutical, medicine, and materials science research.

Finally, we find out the following problems which may become the further topics for the studies in this field:

(i) What are the upper and lower bound of $F$-index in the setting that some graph parameters (diameter, vertex connectivity or edge connectivity, chromatic number, etc.) are fixed? And which molecular structures can reach these maximum or minimum numbers?

(ii) In many engineering applications in chemical, material, medicine, and pharmaceutical field, how is $F$ index used in these fields to test the physical, chemical, biology, and pharmacological properties?

(iii) What is the relationship between $F$-index and other existing degree-based indices (such as atom-bond connectivity index, first and second Zagreb indices, Randic index, Balaban index, Narumi Katayama index, geometric-arithmetic index, sum connectivity index, harmonic index, etc.)?

\section{Competing Interests}

The authors declare that there is no conflict of interests regarding the publication of this paper.

\section{Acknowledgments}

This work was supported in part by the National Natural Science Foundation of China (nos. 11401519 and 61262070). The authors earnestly appreciate all Akbar Ali's warm help earnestly for providing a valuable comment and correcting some mistakes on the computation results.

\section{References}

[1] I. Gutman, "Total $\pi$-electron energy of conjugated molecules with non-bonding molecular orbitals," Zeitschrift fur Naturforschung-Section A: Journal of Physical Sciences, vol. 71, no. 2, pp. 161-164, 2016.

[2] I. Gutman, G. Indulal, and R. Todeschini, "Generalizing the McClelland bounds for total $\pi$-electron energy," Zeitschrift für Naturforschung A, vol. 63, no. 5-6, pp. 280-282, 2008.

[3] I. Gutman, S. Gojak, B. Furtula, S. Radenković, and A. Vodopivec, "Relating total $\pi$-electron energy and resonance energy of benzenoid molecules with Kekulé- and Clarstructure-based parameters," Monatshefte für Chemie, vol. 137, no. 9, pp. 1127-1138, 2006.

[4] I. Gutman, N. Cmiljanović, S. Milosavljević, and S. Radenković, "Effect of non-bonding molecular orbitals on total $\pi$-electron energy," Chemical Physics Letters, vol. 383, no. 1-2, pp. 171-175, 2004.

[5] I. Gutman and K. C. Das, "Estimating the total $\pi$-electron energy," Journal of the Serbian Chemical Society, vol. 78, no. 12, pp. 1925-1933, 2013.

[6] E. L. Angelina, D. J. R. Duarte, and N. M. Peruchena, "Is the decrease of the total electron energy density a covalence indicator in hydrogen and halogen bonds?" Journal of Molecular Modeling, vol. 19, no. 5, pp. 2097-2106, 2013.

[7] L. Türker and I. Gutman, "Iterative estimation of total $\pi$ electron energy," Journal of the Serbian Chemical Society, vol. 70, no. 10, pp. 1193-1197, 2005.

[8] N. C. Jones, D. Field, and J.-P. Ziesel, "Low-energy total electron scattering in the methyl halides $\mathrm{CH}_{3} \mathrm{Cl}, \mathrm{CH}_{3} \mathrm{Br}$ and $\mathrm{CH}_{3} \mathrm{I}$," International Journal of Mass Spectrometry, vol. 277, no. 1-3, pp. 91-95, 2008. 
[9] S. Radenković and I. Gutman, "Total $\pi$-electron energy and Laplacian energy: how far the analogy goes?" Journal of the Serbian Chemical Society, vol. 72, no. 12, pp. 1343-1350, 2007.

[10] M. Peric, I. Gutman, and J. Radic-Peric, “The Hückel total $\pi$ electron energy puzzle," Journal of the Serbian Chemical Society, vol. 71, no. 7, pp. 771-783, 2006.

[11] D. A. Morales, "The total $\pi$-electron energy as a problem of moments: application of the Backus-Gilbert method," Journal of Mathematical Chemistry, vol. 38, no. 3, pp. 389-397, 2005.

[12] S. Markovic, "Approximating total $\pi$-electron energy of phenylenes in terms of spectral moments," Indian Journal of Chemistry Section A-Inorganic Bio-Inorganic Physical Theoretical \& Analytical Chemistry, vol. 42, no. 6, pp. 13041308, 2003.

[13] D. A. Morales, "Systematic search of bounds for total $\pi$-electron energy," International Journal of Quantum Chemistry, vol. 93, no. 1, pp. 20-31, 2003.

[14] B. Furtula and I. Gutman, "A forgotten topological index," Journal of Mathematical Chemistry, vol. 53, no. 4, pp. 1184-1190, 2015.

[15] W. Gao and M. R. Farahani, "Computing the reverse eccentric connectivity index for certain family of nanocone and fullerene structures," Journal of Nanotechnology, vol. 2016, Article ID 3129561, 6 pages, 2016.

[16] W. Gao and W. F. Wang, "Second atom-bond connectivity index of special chemical molecular structures," Journal of Chemistry, vol. 2014, Article ID 906254, 8 pages, 2014.

[17] W. Gao and W. Wang, "The vertex version of weighted Wiener number for bicyclic molecular structures," Computational and Mathematical Methods in Medicine, vol. 2015, Article ID 418106, 10 pages, 2015.

[18] W. Gao, W. F. Wang, and M. R. Farahani, "Topological indices study of molecular structure in anticancer drugs," Journal of Chemistry, vol. 2016, Article ID 3216327, 8 pages, 2016.

[19] W. Gao and M. R. Farahani, "Degree-based indices computation for special chemical molecular structures using edge dividing method," Applied Mathematics and Nonlinear Sciences, vol. 1, no. 1, pp. 99-122, 2016.

[20] F. Yang, X. Wang, M. Li et al., "Templated synthesis of singlewalled carbon nanotubes with specific structure," Accounts of Chemical Research, vol. 49, no. 4, pp. 606-615, 2016.

[21] N. L. Marana, A. R. Albuquerque, F. A. La Porta, E. Longo, and J. R. Sambrano, "Periodic density functional theory study of structural and electronic properties of single-walled zinc oxide and carbon nanotubes," Journal of Solid State Chemistry, vol. 237, pp. 36-47, 2016.

[22] S. N. Li, J. B. Liu, and B. X. Liu, "First principles study of nanostructured $\mathrm{TiS}_{2}$ electrodes for $\mathrm{Na}$ and $\mathrm{Mg}$ ion storage," Journal of Power Sources, vol. 320, pp. 322-331, 2016.

[23] J. A. Bondy and U. S. R. Mutry, Graph Theory, Springer, Berlin, Germany, 2008.

[24] A. Ali, A. A. Bhatti, and Z. Raza, "A note on the zeroth-order general Randić index of cacti and polyomino chains," Iranian Journal of Mathematical Chemistry, vol. 5, pp. 143-152, 2014. 

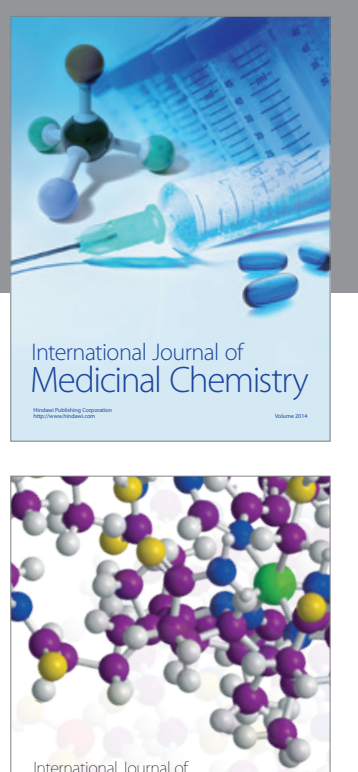

Carbohydrate Chemistry

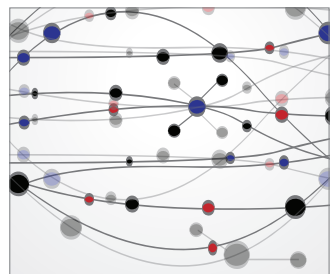

The Scientific World Journal
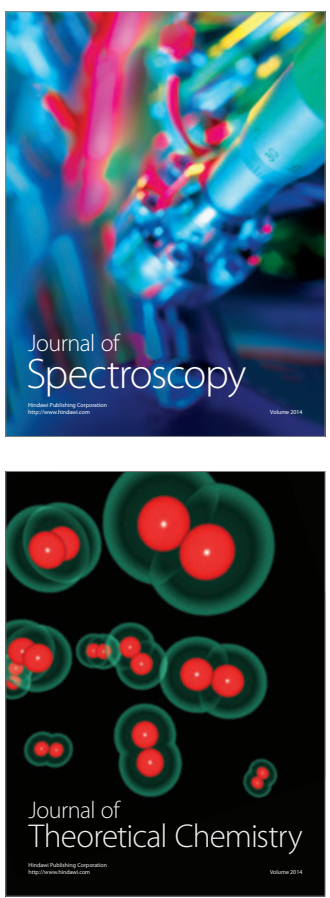
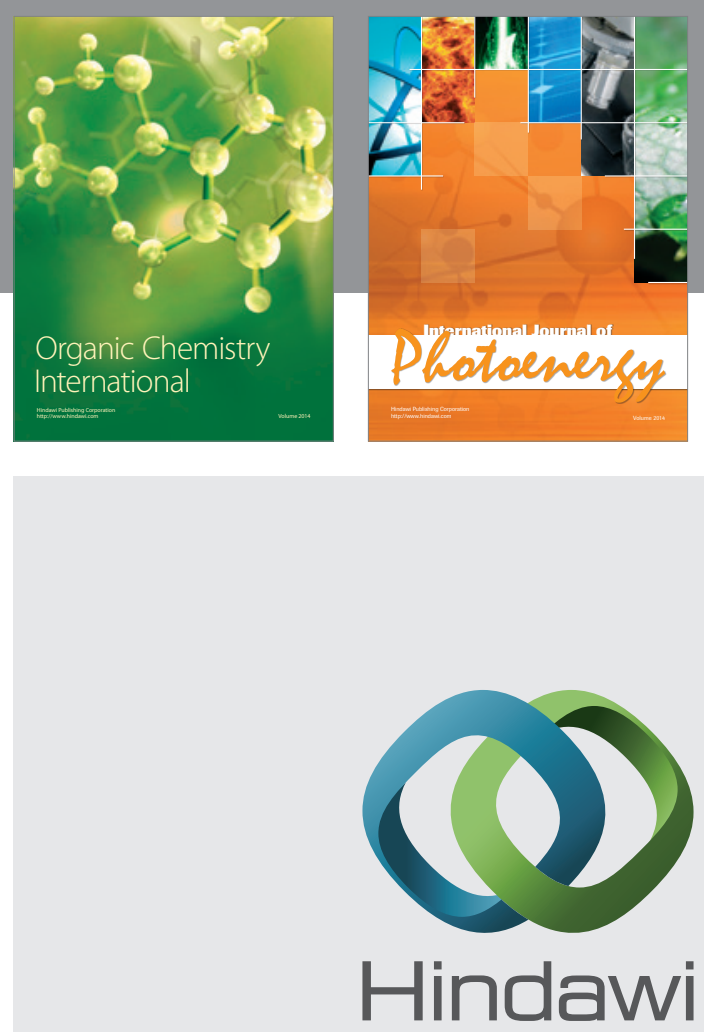

Submit your manuscripts at

http://www.hindawi.com

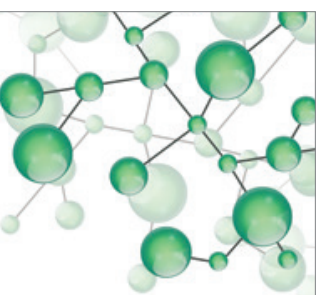

International Journal of

Inorganic Chemistry

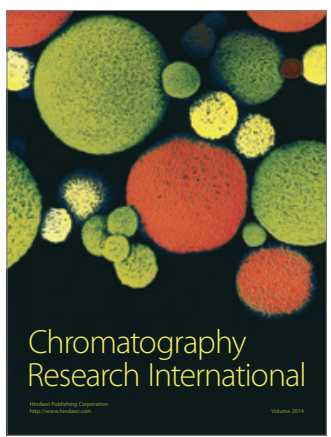

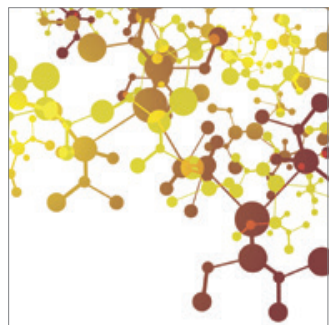

Applied Chemistry
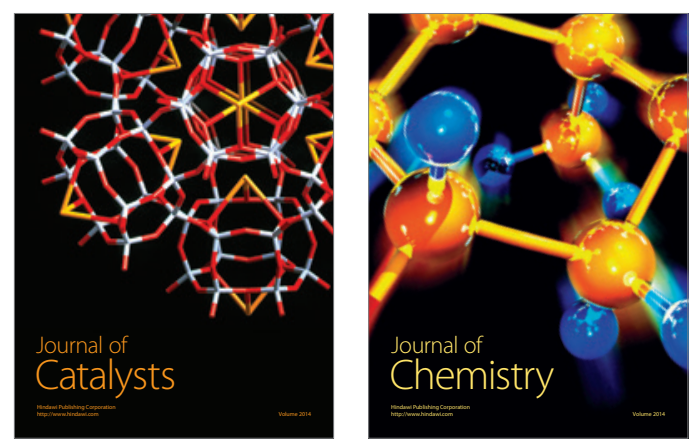
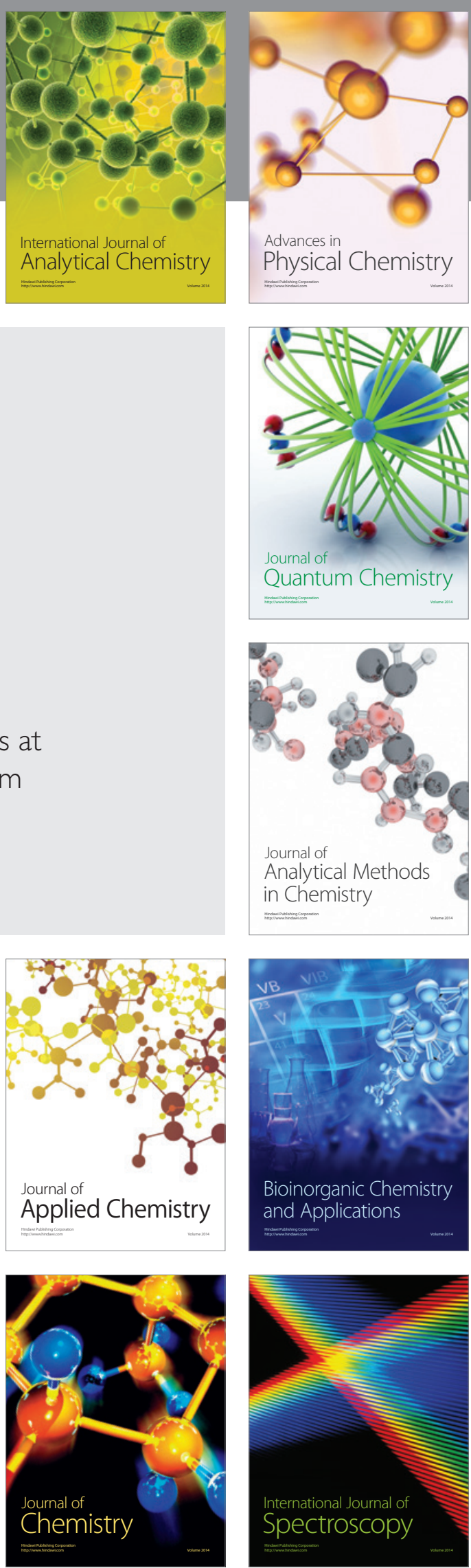